Published in final edited form as:

Lung Cancer. 2012 September ; 77(3): 545-549. doi:10.1016/j.lungcan.2012.04.019.

\title{
Survival among Non-Small Cell Lung Cancer Patients with Poor Performance Status after First Line Chemotherapy
}

\author{
Ramzi G. Salloum, $\mathrm{PhD}^{1}$, Thomas J. Smith, $\mathrm{MD}^{2}$, Gail A. Jensen, $\mathrm{PhD}^{3}$, and Jennifer Elston \\ Lafata, $\mathrm{PhD}^{4}$ \\ ${ }^{1}$ Health Policy and Management, Gillings School of Global Public Health, University of North \\ Carolina at Chapel Hill, Chapel Hill, NC and Center for Health Policy and Health Services \\ Research, Henry Ford Health System, Detroit, MI, USA. ramzi.salloum@unc.edu \\ 2Johns Hopkins Medical Institutes and the Sidney Kimmel Comprehensive Cancer Center, Johns \\ Hopkins University, Baltimore, MD, USA. tsmit136@jhmi.edu \\ ${ }^{3}$ Institute of Gerontology and Department of Economics, Wayne State University, Detroit, MI, \\ USA. gail.jensen@wayne.edu \\ ${ }^{4}$ Social and Behavioral Health and Massey Cancer Center, School of Medicine, Virginia \\ Commonwealth University, Richmond, VA and Center for Health Policy and Health Services \\ Research, Henry Ford Health System, Detroit, MI, USA. jelstonlafat@vcu.edu
}

\begin{abstract}
Background-Performance status (PS) is a commonly used factor in determining the appropriateness for chemotherapy of patients with non-small cell lung cancer (NSCLC). The prevalence of poor PS and impact of chemotherapy on survival among NSCLC patients has not been studied in community populations.
\end{abstract}

Patients and Methods-Insured patients, aged 50+ years, diagnosed with advanced stage NSCLC between 2000 and 2007 were identified via tumor registry $(n=292)$ and linked to electronic medical records, automated medical claims, and Census tract information. A multivariate Cox proportional hazards model was used to determine the factors associated with survival.

Results-Of 292 stage IIIB-IV patients, 82 (28\%) had PS 3 or 4, and 39\% of PS 3-4 patients received first line chemotherapy. Those who received chemotherapy lived 4.8 months compared to 2.4 months for those who did not. Factors associated with a reduced likelihood of death included receipt of chemotherapy (hazard ratio [HR], 0.64), and female gender (HR, 0.71).

Modern chemotherapy may be associated with positive effects on survival for poor PS patients, as for good PS patients. Further trials, especially randomized trials, in this neglected subgroup are indicated.

\footnotetext{
(C) 2012 Elsevier Ireland Ltd. All rights reserved

Address correspondence to: Ramzi G. Salloum 1102F McGavran-Greenberg 135 Dauer Drive Chapel Hill, NC 27599 United States Phone: 919-843-5507 Fax: 919-843-6362 ramzi.salloum@unc.edu.

Publisher's Disclaimer: This is a PDF file of an unedited manuscript that has been accepted for publication. As a service to our customers we are providing this early version of the manuscript. The manuscript will undergo copyediting, typesetting, and review of the resulting proof before it is published in its final citable form. Please note that during the production process errors may be discovered which could affect the content, and all legal disclaimers that apply to the journal pertain.

Conflict of interest statement: None declared.
} 


\section{Keywords}

chemotherapy; non-small cell lung cancer; performance status; survival; advanced stage; guidelines

\section{INTRODUCTION}

Lung cancer is the second most common cancer diagnosed in the United States and the leading cause of cancer-related deaths, with an estimated 221,130 new cases and 156,940 deaths in 2011[1]. Evidence-based treatment guidelines recommend chemotherapy for medically fit patients with unresectable or stage IV non-small cell lung cancer (NSCLC) [25]. A key factor in evaluating the appropriateness of chemotherapy is the patient's performance status (PS). PS is a subjective composite measure used by clinicians to measure functional capacity and the likelihood of adverse events, quality of life, and survival after treatment.

The benefit of chemotherapy for poor PS patients continues to be speculative. Since at least 2004, many oncologists considered chemotherapy appropriate for patients with ECOG PS 2 [6] although the optimal treatment remains controversial, and treatments that worked in patients with PS 0 or 1 do not always translate into success in PS 2 [7]. Most clinical trials purposely have excluded those with poor PS, and existing evidence of limited benefits among poor PS patients derives from only a few small trials [8-10]. Yet, the receipt of chemotherapy among those with poor PS appears to be increasing [11].

The recommendation against chemotherapy in patients with poor PS (ECOG 3 and 4) dates to the early 1980s when PS was found to be a predictor of poor survival, reduced response and worsened toxicity to chemotherapy available at that time $[12,13]$. Newer less toxic drugs, better supportive care, and an emphasis on chemotherapy to improve quality of life versus survival has led to a few Phase II trials in poor PS patients. Leong et al [9] treated 63 PS 3 patients with weekly gemcitabine, docetaxol, or vinorelbine and noted a response rate of 19\%, the same as PS 1-2 patients. Quality of life and breathlessness improved, even in PS 3 patients, and the toxicity of weekly treatment was acceptable. The overall survival was 3.5 months, worse than PS $0-2$ patients. Baka et al [10] found that single agent gemcitabine caused responses in $8 \%$ and stable disease in an additional $16 \%$ of PS 3 patients and may have slightly improved QOL; median survival was $65-83$ days. Furthermore, PS is unavailable through claims data, and thus, no large population studies have been able to study the relationship between PS and outcomes. A recent study, however, examined survival of advanced NSCLC patients in Medicare following chemotherapy [14] using a set of claims based measures to control for a proxy of poor PS. Among a cohort of US community lung cancer patients, we evaluate the effectiveness of modern era chemotherapy treatments on length of survival. Of particular interest is whether the use of chemotherapy has survival advantages among those with poor PS.

\section{METHODS}

\section{Study Population and Setting}

The observational database that includes medical record abstracted PS has been described previously $[11,15]$. Briefly, study patients were identified from among those receiving care from a 900-physician member multispecialty, salaried medical group in southeast Michigan. Patients diagnosed with stage IIIB or IV NSCLC between January 1, 2000 and December 31,2007 who were aged $\geq 50$ years at the time of diagnosis were identified via tumor registry. The medical group, which provides care under both fee-for-service and capitated 
arrangements, staffs 27 primary care clinics throughout Detroit and the surrounding metropolitan area. Study eligible patients were those continuously enrolled in an affiliated health plan (i.e., health maintenance organization) for the one-year period preceding their NSCLC diagnosis for whom we could identify PS in the electronic medical record (EMR) $(N=292)$. No patient received targeted therapies (gefitinib or erlotinib) as none were approved for such use at that time. The study protocol was approved by the medical group's institutional review board.

\section{Analytical Variables}

Two trained chart abstractors reviewed inpatient and outpatient nursing and physician notes available within the patient's EMR from diagnosis until the first notation of death, health plan disenrollment, initiation of chemotherapy, or six months after diagnosis to obtain PS closest to diagnosis date. Performance status was taken directly from the medical chart as recorded by the medical oncologist or oncology nurse. Abstractors recorded the PS documented closest to the diagnosis date since most NSCLC patients start treatment at the time of symptomatic diagnosis and watchful waiting is not recommended by any guideline. If no specific PS was documented, they estimated PS based on medical notes. In most cases, PS was assigned by a medical oncologist (89\%). In cases where patients did not see a medical oncologist, we obtained PS information from records of patient visits to radiation oncologists (5\%) or pulmonologists and primary care providers (5\%). On average PS was captured 23.2 days after diagnosis (standard deviation [SD], 20.5). Of the 292 patients with PS information, 175 (60\%) had a numeric PS (138 using the ECOG scale and 37 using the KPS scale), 44 patients (15\%) had a documented good/poor PS, and the PS for 73 patients (25\%) was extrapolated from EMR notes as described elsewhere [15]. If chemotherapy use was documented in the chart, we considered this "first line" chemotherapy and captured the type of agent used.

Patient age at diagnosis (in years), gender, race, date of diagnosis, stage at diagnosis, and death date were obtained from the tumor registry. In addition to cancer-related information, information regarding other diagnoses in the 12 months preceding diagnosis was obtained from automated claims data and used to construct the Deyo adaptation of the Charlson comorbidity index (CCI) [16]. Claims data were also used to identify initial cancer treatment (i.e., first line chemotherapy, surgery, and/or radiation therapy). Geo-coding, using residential street address and Census tract level data from the 2000 US Census, was used to construct measures of educational achievement and median household income for the neighborhood in which patients resided.

\section{Statistical Analysis}

We used Kaplan Meier estimates to evaluate the survival of patients receiving first line chemotherapy versus those not receiving chemotherapy. Cox proportional hazards analysis was used to evaluate the impact of chemotherapy on survival, adjusting for PS, receipt of surgery and radiation therapy, stage, age, gender, race, CCI, diagnosis year, and geo-coded college graduation rate and median household income. Per the 2009 American Society of Clinical Oncology (ASCO) practice guidelines [17], chemotherapy was recommended for patients with good PS (i.e., PS= 0-2) but not patients with poor PS (i.e., PS=3-4). Earlier ASCO guidelines recommended chemotherapy for patients with $\mathrm{PS}=0-1$ only [2].

\section{Sensitivity Analysis}

In baseline models we included patients with PS $=0-2$ in the good PS group, since many oncologists were giving chemotherapy to PS 2 patients even before 2004 [6]. Alternative models considered PS $=2$ patients with the poor PS group. We tested the two-way interaction between chemotherapy receipt/non-receipt and good/poor PS to determine 
whether the effect of chemotherapy receipt was moderated by PS. We also made the assumption that documented poor PS without a numeric value (i.e. either KPS or ECOG) did not include PS2 patients, but tested the sensitivity of our model using a subsample of only numeric PS. Although the CCI has been shown to be independent of functional status in cancer patients [18], we measured the correlation between PS and the CCI and tested an alternative model that excluded the comorbidity index. All analyses used two-sided tests and were performed using SAS version $9.2 ; P<.05$ was considered statistically significant.

\section{RESULTS \\ Cohort Characteristics}

Cohort characteristics by PS and chemotherapy receipt/non-receipt are presented in Table 1. The cohort was $41 \%$ female, the mean age was 67.6 years (SD, 8.6), and $66 \%$ were white, $31 \%$ black, and $3 \%$ of other races. Of 292 patients, 82 (28\%) had PS 3 or 4 . For 46 of 82 , the PS was only recorded as "poor" and not given a number of 3 or 4 .

\section{Chemotherapy Use}

The rate of first line chemotherapy receipt was $75 \%$ for good PS and $39 \%$ for poor PS patients. Table 2 reports the use of chemotherapy agents by PS. The use of single agent chemotherapy was more common among poor PS patients whereas the use of combination platinum-taxane chemotherapy was more common among good PS patients. The most common regimen used during this time period was carboplatin and paclitaxel (and not single agent gemcitabine or vinorelbine). The use of chemotherapy increased during the years of observation (2000-2007) among both good and poor PS patients, yet no noticeable trends in the use of specific agents were observed (data not shown). Of the 10 patients with recorded PS 4, only 1 received chemotherapy; of 26 with recorded PS 3, 11 (24\%) received chemotherapy.

\section{Survival}

The median length of survival among good PS patients who received first line chemotherapy was 10.8 months compared to 5.8 months for those without chemotherapy. Poor PS patients who received chemotherapy had a median length of survival of 4.8 months compared with 2.4 months for those without chemotherapy. Figure 1 shows survival estimates for patients with good PS (i.e. ECOG 0-2), stratified by choice of chemotherapy receipt or non-receipt. Figure 2 shows similar estimates for patients with poor PS (i.e. ECOG 3-4). Multivariate Cox regression analysis (Table 3 ) found that receipt of chemotherapy (hazard ratio [HR], $0.64)$ and were significantly associated with a lower risk of death. Female gender (HR, 0.71), good PS (HR, 0.50), and diagnosis in or after 2003 (HR, 0.75) were also significant factors that lowered the risk of death, whereas stage IV diagnosis (HR, 1.67) was significantly associated with a higher risk of death. The two-way interaction between chemotherapy receipt/non-receipt and good/poor PS was non-significant, implying that the increase in survival associated with chemotherapy receipt did not differ by PS. Results from the model in which patients with PS $=2(n=37)$ were aligned with the poor PS group; from the model that excludes the CCI; and the model limited to numeric PS were neither statistically nor substantively different (data not shown). We found the Pearson correlation between PS and CCI to be 0.32 .

\section{DISCUSSION}

To our knowledge this is the first community-based study of the prevalence of poor ( 3 and 4) PS in NSCLC patients, and the effectiveness of chemotherapy on survival among a modern U.S. lung cancer cohort with both good and poor PS patients. Findings here 
illustrate the significant and positive association of first line chemotherapy on the survival of NSCLC patients, regardless of PS. Our results are comparable to the benefit seen in the two trials published that show PS 3 patients tolerate chemotherapy, do not have excess toxicity, and have improved quality of life and symptoms $[8,9]$. Our survival results are similar to those from recent community based oncology studies of NSCLC chemotherapy [19]. The most recent showed a median survival of 9-10 months for PS 0-1 patients, but only 7\% of 1409 treated patients had documented PS of 2 or 3 [20]; as in our study, PS 2 or 3 was a significant predictor for worse survival, (HR 2.63 to 4.57 for PS of 2 to $3 ; P<.001$ ). Our results (HR 0.67 for receipt of chemotherapy and survival) are also very consistent with the recent update of the Cochrane Database showing a significant benefit of chemotherapy (HR $0.77 ; 95 \%$ CI 0.71 to $0.83, P<.001)$ [21].

The main limitation of the current study is the potential for selection bias as there may be other factors beyond PS and those controlled in the model which physicians and patients consider when weighing the possible benefits and harms of chemotherapy. Another limitation is the subjective and biased nature of PS that leads to patients interested in chemotherapy possibly having a higher likelihood of scoring a good PS. Further, even though we control for a comorbidity index, we are unable to fully distinguish between patients with disease related poor PS vs. longstanding poor PS. Also, this is nearly all PS 3 patients, because only 1 of 10 known recorded PS 4 patients received chemotherapy. Nonetheless, our finding of a survival benefit among NSCLC patients receiving chemotherapy, regardless of PS, is consistent with findings from recent, small clinical trials [8-10]. The survival of this cohort of poor PS patients, 2 to 5 months, is consistent with those trials, and the possible 3-month added survival is consistent with other studies of first line chemotherapy [17]. Another limitation of our study is the inability to determine the doses and full lines of chemotherapy that were received, the toxicities, and response rates. Finally, our results are based on a sample of patients with documented PS and may not be generalizable to patients without PS documented in the EMR.

Treatment of patients with newer targeted therapies despite poor PS has been shown to have some benefit with acceptable toxicity [8] and it is possible that well-tolerated chemotherapy could have the same effect [22]. These treatments could be expensive and increase end of life care costs, or actually reduce symptom burden and increase survival, so this issue will be at the forefront of rational resource allocation [23]. Given the prevalence of poor PS in community cohorts (34\% classified as PS $2-4$ by physicians, $48 \%$ self-classified as PS $2-4$ [24], and one third with database-derived poor PS [14]) and the limited number of trials enrolling poor PS patients, research is needed to confirm these findings in a larger and more diverse sample or in a randomized controlled trial where the only difference is receipt of chemotherapy, and all patients are offered reasonable and defined best supportive care [25]. The evaluation of treatment of poor performance patients will become especially important with the availability of costly targeted drugs that have fewer toxicities and some benefit.

\section{Acknowledgments}

This work was based in part on a doctoral dissertation submitted by Ramzi Salloum to the Department of Economics at Wayne State University. We thank Elizabeth Dobie and Nonna Akkerman for their assistance with data acquisition. This work was supported by the Fund for Henry Ford and the National Cancer Institute at the National Institutes of Health under [grant NIH R01 CA114204]. Dr. Salloum was supported by the Student Award Program from the Blue Cross and Blue Shield of Michigan Foundation under [grant 1705.SAP] and by the Cancer Care Quality Training Program through the National Cancer Institute at the National Institutes of Health under [grant R25 CA116339]. Dr. Smith was supported by the American Cancer Society [grant PEP-10-174-01], and the National Cancer Institute at the National Institutes of Health under [grants R01 CA116227, R01 CA106370]. 


\section{References}

[1]. American Cancer Society. Cancer Facts and Figures. 2011.

[2]. Clinical practice guidelines for the treatment of unresectable non-small-cell lung cancer. Adopted on May 16, 1997 by the American Society of Clinical Oncology. J Clin Oncol. 1997; 15:29963018. [PubMed: 9256144]

[3]. National Cancer Institute. [accessed May 11, 2011] Physician Data Query Cancer Information Summaries. Available from URL: http://www.nci.nih.gov/cancerinfo/pdq/treatment/non-small-cell-lung//healthprofessional/

[4]. National Comprehensive Cancer Network. [accessed January 20, 2011] Non-Small Cell Lung Cancer. Practice Guidelines in Oncology. Available from URL: http://www.ncen.org

[5]. Pfister DG, Johnson DH, Azzoli CG, et al. American society of clinical oncology treatment of unresectable non-small-cell lung cancer guideline: Update 2003. J Clin Oncol. 2004; 22:330 353. [PubMed: 14691125]

[6]. Lilenbaum RC. Treatment of advanced non-small-cell lung cancer in special populations. Oncology. 2004; 18:1321-5. discussion 1326, 1329-33. [PubMed: 15526837]

[7]. Lilenbaum R, Wang X, Gu L, Kirshner J, Lerro K, Vokes E. Randomized phase II trial of docetaxel plus cetuximab or docetaxel plus bortezomib in patients with advanced non-small-cell lung cancer and a performance status of 2: CALGB 30402. J Clin Oncol. 2009; 27:4487-4491. [PubMed: 19704058]

[8]. Liu S, Wang D, Chen B, Wang Y, Zhao W, Wu J. The safety and efficacy of EGFR TKIs monotherapy versus single-agent chemotherapy using third-generation cytotoxics as the first-line treatment for patients with advanced non-small cell lung cancer and poor performance status. Lung Cancer. 2011; 73:203-210. [PubMed: 21211862]

[9]. Leong SS, Toh CK, Lim WT, et al. A randomized phase II trial of single-agent gemcitabine, vinorelbine, or docetaxel in patients with advanced non-small cell lung cancer who have poor performance status and/or are elderly. J Thorac Oncol. 2007; 2:230-236. [PubMed: 17410046]

[10]. Baka S, Ashcroft L, Anderson H, et al. Randomized phase II study of two gemcitabine schedules for patients with impaired performance status (karnofsky performance status $</=70$ ) and advanced non-small-cell lung cancer. J Clin Oncol. 2005; 23:2136-2144. [PubMed: 15713598]

[11]. Salloum RG, Smith TJ, Jensen GA, Lafata JE. Factors associated with adherence to chemotherapy guidelines in patients with non-small cell lung cancer. Lung Cancer. 2012; 75:255-260. [PubMed: 21816502]

[12]. Stanley KE. Prognostic factors for survival in patients with inoperable lung cancer. J Natl Cancer Inst. 1980; 65:25-32. [PubMed: 6930515]

[13]. Pater JL, Loeb M. Nonanatomic prognostic factors in carcinoma of the lung: A multivariate analysis. Cancer. 1982; 50:326-331. [PubMed: 7083139]

[14]. Davidoff AJ, Tang M, Seal B, Edelman MJ. Chemotherapy and survival benefit in elderly patients with advanced non-small-cell lung cancer. J Clin Oncol. 2010; 28:2191-2197. [PubMed: 20351329]

[15]. Salloum RG, Smith TJ, Jensen GA, Lafata JE. Using claims-based measures to predict performance status score in patients with lung cancer. Cancer. 2011; 117:1038-1048. [PubMed: 20957722]

[16]. Deyo RA, Cherkin DC, Ciol MA. Adapting a clinical comorbidity index for use with ICD-9-CM administrative databases. J Clin Epidemiol. 1992; 45:613-619. [PubMed: 1607900]

[17]. Azzoli CG, Baker S Jr, Temin S, et al. American society of clinical oncology clinical practice guideline update on chemotherapy for stage IV non-small-cell lung cancer. J Clin Oncol. 2009; 27:6251-6266. [PubMed: 19917871]

[18]. Extermann M, Overcash J, Lyman GH, Parr J, Balducci L. Comorbidity and functional status are independent in older cancer patients. J Clin Oncol. 1998; 16:1582-1587. [PubMed: 9552069]

[19]. Saito AM, Landrum MB, Neville BA, Ayanian JZ, Earle CC. The effect on survival of continuing chemotherapy to near death. BMC Palliat Care. 2011; 10:14. [PubMed: 21936940] 
[20]. Neubauer MA, Hoverman JR, Kolodziej M, et al. Cost effectiveness of evidence-based treatment guidelines for the treatment of non-small-cell lung cancer in the community setting. J Oncol Pract. 2010; 6:12-18. [PubMed: 20539725]

[21]. Non-Small Cell Lung Cancer Collaborative Group. Chemotherapy and supportive care versus supportive care alone for advanced non-small cell lung cancer. Cochrane Database Syst Rev. 2010; (5):CD007309. [PubMed: 20464750]

[22]. Langer CJ. Clinical evidence on the undertreatment of older and poor performance patients who have advanced non-small-cell lung cancer: Is there a role for targeted therapy in these cohorts? Clin Lung Cancer. 2011

[23]. Smith TJ, Hillner BE. Bending the cost curve in cancer care. N Engl J Med. 2011; 364:20602065. [PubMed: 21612477]

[24]. Lilenbaum RC, Cashy J, Hensing TA, Young S, Cella D. Prevalence of poor performance status in lung cancer patients: Implications for research. J Thorac Oncol. 2008; 3:125-129. [PubMed: 18303431]

[25]. Jack B, Boland A, Dickson R, Stevenson J, McLeod C. Best supportive care in lung cancer trials is inadequately described: A systematic review. Eur J Cancer Care. 2010; 19:293-301. 


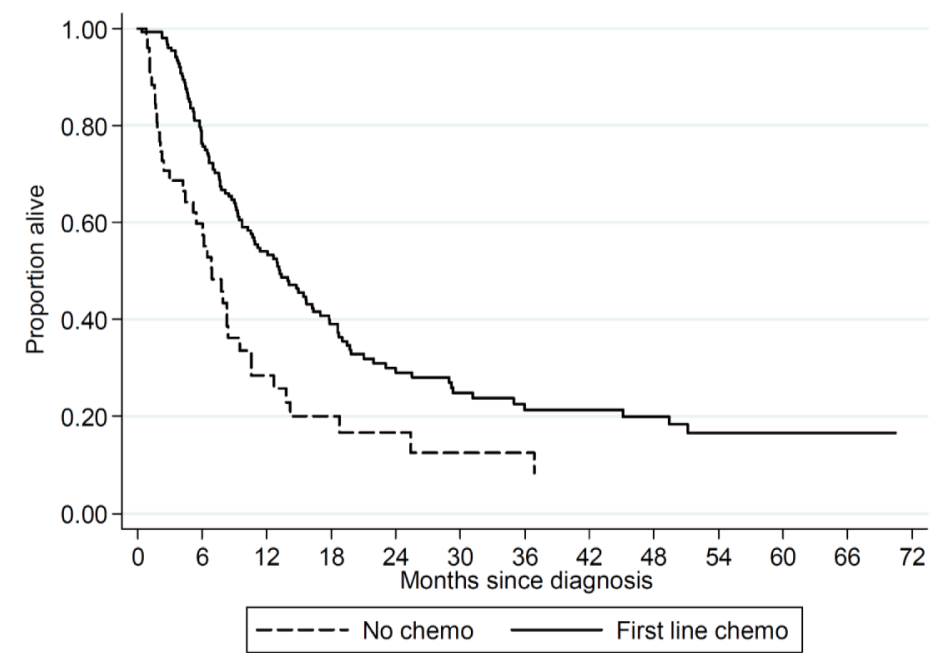

Figure 1.

Survival of advanced non-small cell lung cancer patients, ECOG performance status 0-2, with first line chemotherapy (solid line) vs. no chemotherapy (dashed line). 


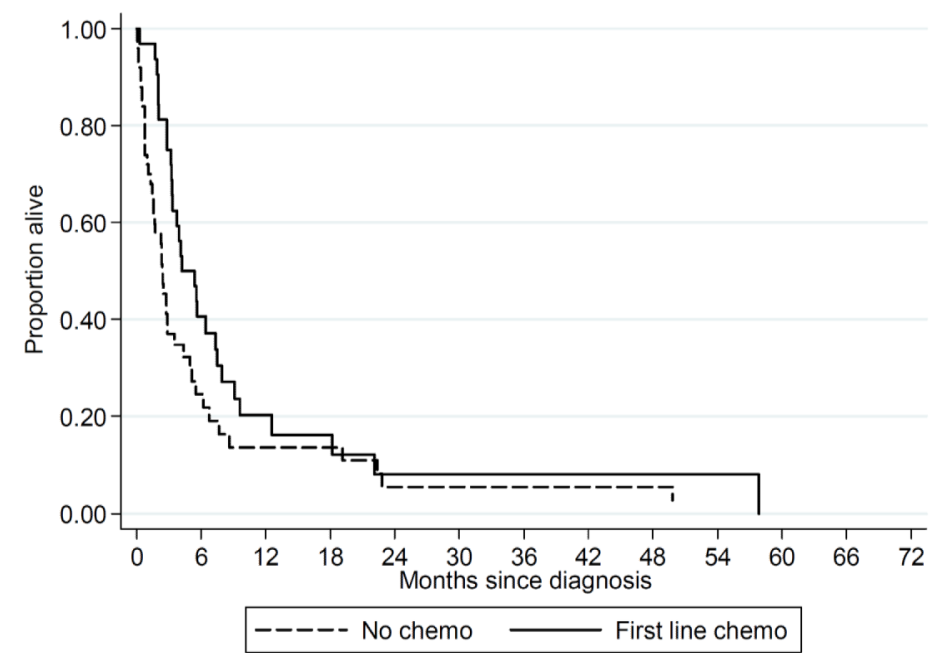

Figure 2.

Survival of advanced non-small cell lung cancer patients, ECOG performance status 3-4, with first line chemotherapy (solid line) vs. no chemotherapy (dashed line). 


\section{Table 1}

Cohort characteristics and survival for patients diagnosed with advanced stage non-small cell lung cancer between 2000 and 2007, by performance status (PS) and choice of chemotherapy receipt or non-receipt, $(N=$ 292)

\begin{tabular}{|c|c|c|c|c|}
\hline & \multicolumn{2}{|c|}{$\operatorname{Good~PS}^{1}(N=210)$} & \multicolumn{2}{|c|}{ 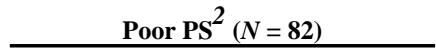 } \\
\hline & Chemo $(N=158)$ & No Chemo $(N=52)$ & Chemo $(N=32)$ & No Chemo $(N=50)$ \\
\hline \multicolumn{5}{|l|}{ Demographic Characteristics } \\
\hline Mean age at diagnosis (SD) & $65.6(8.1)^{3}$ & $71.0(9.5)^{\ddagger}$ & $67.3(9.1)^{4}$ & $70.7(6.7)^{\S}$ \\
\hline \multicolumn{5}{|l|}{ Gender $(\%)$} \\
\hline Female & 42 & 46 & 28 & 40 \\
\hline Male & 58 & 54 & 72 & 60 \\
\hline \multicolumn{5}{|l|}{ Race $(\%)$} \\
\hline Black & 29 & 29 & 31 & 38 \\
\hline White & 70 & 65 & 69 & 56 \\
\hline Other & 1 & 6 & - & 6 \\
\hline \multicolumn{5}{|l|}{ Clinical Characteristics } \\
\hline \multicolumn{5}{|l|}{ AJCC stage (\%) } \\
\hline IIIB & 30 & 29 & 22 & 24 \\
\hline IV & 70 & 71 & 78 & 76 \\
\hline Average Charlson comorbidity index (SD) & $1.0(1.4)$ & $1.4(1.4)$ & $2.0(2.0)$ & $2.9(3.4)$ \\
\hline \multicolumn{5}{|l|}{ Neighborhood Socioeconomic Characteristics } \\
\hline Pct with college degree (SD) & $7.4(6.5)$ & $6.0(5.3)$ & $6.9(5.9)$ & $6.4(4.4)$ \\
\hline Median household income in $\$ 1000$ s (SD) & $50.7(23.7)$ & $46.2(19.8)$ & $52.3(28.9)$ & $43.7(17.1)$ \\
\hline \multicolumn{5}{|l|}{ Treatment(s) Received } \\
\hline No treatment $(\%)$ & - & 54 & - & 64 \\
\hline Surgery only $(\%)$ & - & - & - & - \\
\hline Radiation therapy only (\%) & - & 46 & - & 36 \\
\hline Chemotherapy only (\%) & 17 & - & 34 & - \\
\hline Surgery + radiation therapy $(\%)$ & - & - & - & - \\
\hline Surgery + chemotherapy $(\%)$ & 8 & - & - & - \\
\hline Radiation + chemotherapy (\%) & 62 & - & 66 & - \\
\hline Surgery + radiation + chemotherapy $(\%)$ & 13 & - & - & - \\
\hline \multicolumn{5}{|l|}{ Survival } \\
\hline Median survival time in months & $10.8^{\ddagger}$ & $5.8^{\ddagger}$ & $4.8^{\S}$ & $2.4^{\S}$ \\
\hline Died by end of study period (\%) & 68.4 & 75.0 & 90.6 & 88.0 \\
\hline \multicolumn{5}{|l|}{${ }^{1}$ Good PS: ECOG 0-2 } \\
\hline \multicolumn{5}{|l|}{$2^{2}$ Poor PS: ECOG $>2$} \\
\hline
\end{tabular}


Table 2

Use of first line chemotherapy agents by performance status

\begin{tabular}{lrr}
\hline Choice of first line chemotherapy & Good PS ECOG: 0-2 & Poor PS ECOG: 3-4 \\
\hline No chemotherapy $(N)$ & 52 & 50 \\
First line chemotherapy $(N)$ & 158 & 32 \\
Single agent $(\%)$ & 18 & 53 \\
Platinum + non-taxane (\%) & 33 & 25 \\
Platinum + taxane $(\%)$ & 49 & 22 \\
\hline
\end{tabular}




\section{Table 3}

Multivariate Cox proportional hazards model of survival for patients diagnosed with advanced stage non-small cell lung cancer between 2000 and $2007(N=292)$

\begin{tabular}{lccc}
\hline Patient Factors & Parameter & Hazard Ratio & $\boldsymbol{P}$ Value \\
\hline Treatment Received & & & \\
$\quad$ Chemotherapy & -0.44 & 0.64 & 0.01 \\
Patient Demographics & & & \\
$\quad$ Age at diagnosis (years) & 0.01 & 1.01 & 0.23 \\
$\quad$ Gender = female & -0.34 & 0.71 & 0.02 \\
$\quad$ Race = white & 0.16 & 1.17 & 0.36 \\
Clinical Characteristics & & & \\
$\quad$ Performance status = good & & & \\
$\quad$ Stage IV at diagnosis & -0.69 & 0.50 & $<.01$ \\
$\quad$ Charlson comorbidity index & 0.51 & 1.67 & $<.01$ \\
Socioeconomic Characteristics & 0.05 & 1.06 & 0.16 \\
$\quad$ College degree & & & \\
$\quad$ Median income $(\$ 1000 s)$ & -0.01 & 0.94 & 0.17 \\
Guidelines & & & \\
$\quad$ Year of diagnosis > 2003 & -0.29 & 0.75 & 0.05 \\
Model Characteristics & & & \\
Likelihood ratio, $\chi^{2}(10)$ & 80.7 & & \\
$\quad$ Probability > $\chi^{2}$ & $<0.001$ & & \\
\hline
\end{tabular}

* Good PS: ECOG 0-2; Poor PS: ECOG >2. 